\title{
Co oxidation state at LSC-YSZ interface in model solid oxide electrochemical cell
}

\author{
Fabrizio Gaulandris ${ }^{\mathrm{a}}$, Luise Theil Kuhn ${ }^{\mathrm{a}}$, Jakob Birkedal Wagner ${ }^{\mathrm{b}}$, Simone Sanna ${ }^{\mathrm{a}, 1}$, Søren \\ Bredmose Simonsen ${ }^{\text {a, }}$, \\ ${ }^{\text {a }}$ DTU Energy, Technical University of Denmark, Fysikvej, DK-2800 Kgs. Lyngby, Denmark \\ ${ }^{\mathrm{b}}$ DTU Nanolab, Technical University of Denmark, Fysikvej, DK-2800 Kgs. Lyngby, Denmark
}

\section{A R T I C L E I N F O}

\section{Keywords:}

SOFC

SOEC

LSC

YSZ

Environmental STEM

EELS

\begin{abstract}
A B S T R A C T
Oxidation states of cations in a perovskite structure play an important role for conductivity in solid oxide electrochemical cells. For bulk materials oxidation states can be calculated for specific conditions, but is not well understood for micro-/nanostructured materials and at interfaces between materials. We present a fundamental study of interfaces between $\mathrm{La}_{0.6} \mathrm{Sr}_{0.4} \mathrm{CoO}_{3-\delta}$ (LSC) and yttria-stabilized zirconia (YSZ) in symmetric model solid oxide electrochemical cells. Nanoscale morphology as well as the chemical state of the LSC are investigated by scanning transmission electron microscopy (STEM) and electron energy loss spectroscopy (EELS). The experiments were performed in situ at temperatures up to $600{ }^{\circ} \mathrm{C}$ in high vacuum (ca. $10^{-7}$ mbar) and in 2 mbar oxygen. The measured LSC Co oxidation at room temperature is lower than that expected for bulk LSC, indicating a high oxygen vacancy density and possibly high ionic conductivity. However, the Co oxidation state increases with increasing temperature, both in oxygen and in vacuum. The results shows that the Co oxidation state approach that expected for bulk LSC for typical solid oxide fuel or electrolysis cell operation temperatures.
\end{abstract}

\section{Introduction}

The mixed ionic and electronic conductor perovskites $\mathrm{La}_{0.6} \mathrm{Sr}_{0.4}$ $\mathrm{CoO}_{3-\delta}$ (LSC) and $\mathrm{La}_{0.6} \mathrm{Sr}_{0.4} \mathrm{Co}_{0.2} \mathrm{Fe}_{0.8} \mathrm{O}_{3-\delta}$ (LSCF) have recently received increasing attention as oxygen electrodes in solid oxide electrolysis and fuel cells (SOEC/SOFC) [1-5]. This is partly due to a relatively high conductivity at low operation temperatures $\left(500-700{ }^{\circ} \mathrm{C}\right)$ compared to the typical operation temperature of ca. $850{ }^{\circ} \mathrm{C}$ for state of the art SOECs/SOFCs $[2,4,5]$. The conductivity can be improved further by combining LSC or LSCF with other oxides such as gadolinia-doped ceria (CGO) [1], for example as multi-layered structures [3]. Nano-structuring appears to play a significant role for the enhanced conductivity [3], and the electrochemical properties are sensitive to the oxide composition and microstructure at interfaces [1].

In an SOEC/SOFC, the oxygen electrode will share an interface with the electrolyte. Yttria-stabilized zirconia (YSZ) is a state of the art electrolyte in SOECs/SOFCs due to high ionic conductivity at elevated temperatures and very low electronic conductivity.

At elevated temperatures segregation can take place across the LSCYSZ interface and results in formation of $\mathrm{La}_{2} \mathrm{Zr}_{2} \mathrm{O}_{7}$ [6]. The thermodynamic stability of pure LSC and its Co oxidation states, have been widely studied at different conditions, mainly for bulk samples, and the relation of the oxidation states to temperature and oxygen pressure have been described [7-10]. The oxygen vacancy concentration is related to the fraction of the transition metal mixed valence. The amount of oxygen vacancies, $\delta$, in for example $\mathrm{La}_{0.6} \mathrm{Sr}_{0.4} \mathrm{CoO}_{3-\delta}$, increases with decreasing oxygen partial pressure and the increase of temperature [11]. The average valence can be calculated for different temperatures, oxygen partial pressures and percentage of strontium doping, and is for example $\mathrm{Co}^{3+}$ for $\mathrm{La}_{0.6} \mathrm{Sr}_{0.4} \mathrm{CoO}_{3-\delta}$ when the partial pressure of oxygen is $10^{-4}$ atm, and the temperature $800{ }^{\circ} \mathrm{C}[12,13]$.

For a perovskite, the cation oxidation state can vary from bulk to grain boundary [14,15], and further it is not well understood how LSC at or near an LSC-YSZ interface is influenced by the presence of YSZ. In

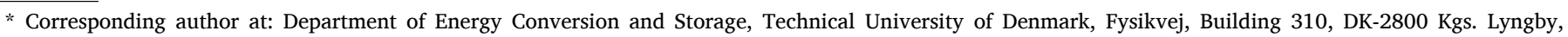
Denmark.

E-mail address: sobrs@dtu.dk (S.B. Simonsen).

1 Present address: Dipartimento di Ingegneria Civile e Ingegneria Informatica and CNR-SPIN, Università di Roma Tor Vergata, Via del Politecnico 1, I-00133 Roma, Italy.
} 
particular, how such an interface influences the stability and oxidation states at different temperatures and varying oxygen pressure.

Information of oxidation states is available from electron energy-loss spectroscopy (EELS). The $\mathrm{L}_{23}$ or $\mathrm{M}_{45}$ edges of transition metals have two characteristic peaks called white lines. The intensity ratio of the white lines depends of the number of electrons in the final $3 \mathrm{~d}$ or $4 \mathrm{f}$ state and scales for some metals, such as Co, with the oxidation state [16]. Measurements of the Co $\mathrm{L}_{23}$ white line ratio is therefore one route for obtaining the Co oxidation state.

An alternative route, suggested by P. Müller et al. [17] is to measure relative shifts of the white line peak position. They managed to correlate the measured energy difference between the $\mathrm{L}_{2}$ and $\mathrm{L}_{3}$ white lines of Co to a change in Co oxidation state [17]. This method is not as wellestablished as using the white line ratio, and Müller et al. comments that "one has to accept at present that the expected dependence of the Co-valence state on the white-line distance is unknown which limits the accuracy of the method." [17].

Compared to spatially averaging methods such as XPS or EXAFS, much higher spatial resolution $(<1 \mathrm{~nm})$ is possible to obtain by combining EELS with scanning transmission electron microscopy (STEM) [16]. By using an environmental STEM (ESTEM) information about the influence of reactive gasses around the interface is accessible.

Here we present a study of LSC-YSZ interfaces in a symmetric model SOEC/SOFC. Temperature-dependent nanoscale changes are characterized by environmental STEM. To investigate the possible influence of oxygen, the environmental STEM experiments were carried out both in vacuum and in $\mathrm{O}_{2}$. Segregation and intermixing across the LSC-YSZ interface as well as the chemical state of the LSC were investigated by EELS. The analysis particularly focus on the Co oxidation state.

\section{Materials and methods}

\subsection{Sample preparation}

A symmetric model solid oxide fuel/electrolysis cell was prepared by pulsed laser deposition (PLD). A substrate of $1 \%$ Niobium doped $\mathrm{SrTiO}_{3}$ $\delta$ (STN) single crystal (growth direction (100) was used. On top of the STN, $300 \mathrm{~nm}$ of $\mathrm{La}_{0.6} \mathrm{Sr}_{0.4} \mathrm{CoO}_{3-\delta}$ (LSC), $300 \mathrm{~nm}$ of $\mathrm{ZrO}_{2}: 8 \% \mathrm{~mol} \mathrm{Y}_{2} \mathrm{O}_{3}$ (8YSZ), and finally $300 \mathrm{~nm}$ of $\mathrm{La}_{0.6} \mathrm{Sr}_{0.4} \mathrm{CoO}_{3-\delta}$ (LSC) was deposited. The LSC/YSZ/LSC layers were grown using a multi-target carousel. The film thicknesses were chosen in order to avoid pinholes. The PLD chamber was evacuated to a base vacuum of $1 \times 10^{-8}$ mbar before the deposition. During the deposition the substrate temperature was $450{ }^{\circ} \mathrm{C}$ and oxygen partial pressure was $10^{-4}$ mbar. A KrF excimer laser (Coherent Lambda Physik $\mathrm{GmbH}$ ) with a wavelength of $248 \mathrm{~nm}$ and pulse duration of $25 \mathrm{~ns}$ was focused on the target. The laser energy density was kept constant at $4 \mathrm{~J} \mathrm{~cm}^{-2}$ with a repetition rate of $30 \mathrm{~Hz}$. The deposition rate for LSC and YSZ was $0.01 \mathrm{~nm}$ per pulse. These PLD growth conditions hinder the formation of secondary phases [18].

\subsection{Environmental STEM-EELS}

Two STEM specimens were prepared from the same symmetric model cell by thinning with a focused ion beam (FIB) using a FIB-SEM Zeiss Crossbeam 1540XB equipped with a field-emission gun. The specimens were attached by platinum deposition to a molybdenum TEM grid for the experiment conducted in vacuum and to a stainless steel TEM grid for the experiment conducted in oxygen.

Two different microscopes, both with a field-emission gun and a Gatan imaging filter (GIF) for EELS observation were used at an accelerating voltage of $300 \mathrm{kV}$. An environmental TEM (ETEM) Titan 80-300 (FEI) equipped with a differential pumping system and an image aberration corrector [19] was used for the experiment conducted in oxygen. A Titan 80-300ST TEM with a probe aberration corrector was used for the experiment conducted in vacuum. Both microscopes where operated in high angle annular dark-field (HAADF) STEM mode. The camera length was $48 \mathrm{~mm}$, the EELS entrance aperture $2.5 \mathrm{~mm}$, convergence and collection semi-angles are $18 \mathrm{mrad}$ and $8 \mathrm{mrad}$, respectively. A GIF was used in combination with STEM to record spectrum images during specimen heating. A Gatan double tilt heating TEM holder was used to heat the specimen in both in situ experiments.

Two heating experiments were conducted, one in high vacuum (ca. $10^{-7}$ mbar) and one in 2 mbar of $\mathrm{O}_{2}$ (AGA 6.0). It should be noticed that this is a low gas pressure compared an oxygen partial pressure of maybe a factor of 100 higher in the oxygen electrode side of an industrial type solid oxide cell. This is due to a limitation of the Environmental TEM which only allow for gas pressures of a few mbar. The present results may therefore show trends in the material behavior, while the magnitude of the effects can be more dramatic in the industrial cells. In both experiments, temperatures were ramped in steps of $100^{\circ} \mathrm{C}$ by $10^{\circ} \mathrm{C} / \mathrm{min}$ from room temperature to $700{ }^{\circ} \mathrm{C}$. At each step, the temperature was kept constant for ca. 120 min to record STEM spectrum images and to stabilize the system from thermal drift. The energy resolution in both microscopes was ca. $1.2 \mathrm{eV}$. A dispersion of $0.5 \mathrm{eV} / \mathrm{channel}$ was applied.

\subsection{EELS data analysis}

In order to compensate for energy drift, a spectrum image alignment by peak (option available in the Gatan's Digital Micrograph ${ }^{\mathrm{TM}}$ software) was carried out using the onset of the $\mathrm{O}-\mathrm{K}$ edge, since this edge was present in all the oxides under analysis.

Fig. 1a presents an example of a spectrum image where each pixel includes an EELS spectrum. To increase the signal-to-noise ratio, the sum
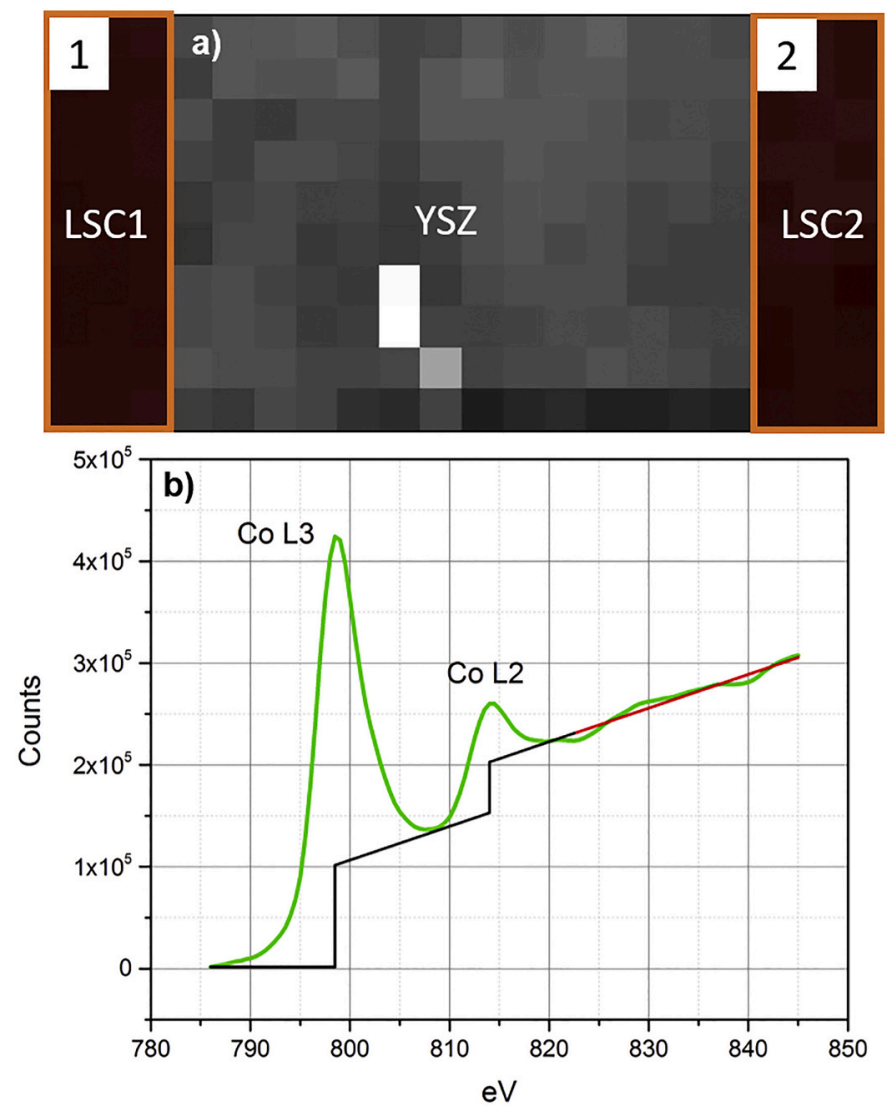

Fig. 1. a) example of a typical spectrum image map obtained from the specimen. Each pixel contains an EELS spectrum. The orange boxes represent the number of pixels averaged to obtain the spectrum used for the calculation of the intensity ratio of Co white lines. b) example of a typical extracted spectrum (green curve). A step function was fitted (black line), to remove the background intensity of the peaks. The slope of the step function was estimated fitting the spectrum after the Co $\mathrm{L}_{2}$ edge (red line). 
of spectra from more pixels were used in the analyses, for example, all pixels in the orange boxes where used for white line ratio analysis (Fig. 1a).

Fig. 1b presents the sharp Co- $\mathrm{L}_{3}$ and $\mathrm{L}_{2}$ edges, the so-called white lines. The intensity ratio of the white lines was estimated using the method from Pearson et al. [20]. First, the power law fitted background was removed and the remaining background intensity under the peaks was modelled with a double step function dispatched in Fig. 1b, black line. The ratio of the steps was 2:1 in accordance with the multiplicity of the initial state (four $2 \mathrm{p}_{3 / 2}$ electrons and $2 \mathrm{p}_{1 / 2}$ electrons). The slope of the step function was estimated by fitting the post Co- $\mathrm{L}_{2}$ edge spectrum (red line), before the influence from the forthcoming La- $\mathrm{M}_{4}$ edge. The intensity of the Co edges was calculated as the difference between the spectrum and the step function.

Multiple scattering effects could unfortunately not be removed by Fourier ratio deconvolution [16] because low-loss spectra were not acquired in order to save time in the relatively long in situ experiments. The absolute values of the white line ratio are therefore not exact and the analysis will emphasize the relative changes observed.

\section{Results}

Fig. 2 presents a series of environmental HAADF-STEM images recorded at the temperatures $\mathrm{RT}, 200{ }^{\circ} \mathrm{C}, 400{ }^{\circ} \mathrm{C}$ and $600{ }^{\circ} \mathrm{C}$ in oxygen ( 2 mbar) and in vacuum (ca. $10^{-7} \mathrm{mbar}$ ). In each image from right to left, the first LSC layer deposited on the STN substrate (LSC1), YSZ and the second LSC layer deposited on YSZ (LSC2) is observed.

The image contrast reveal the three layers with YSZ being slightly darker than the LSC layers. The interfaces between each layers are seen as sharp vertical lines. On top of this, a number of bright regions are observed in all layers, but mostly pronounced in the YSZ and for the sample in oxygen. The shape, size and position of these bright regions change from image to image. We interpret these features not to indicate morphological changes to the LSC and YSZ, but to indicate dislocations, stacking faults and possibly grain boundaries in columnar structures $[21,22]$. The changes in the appearance of the features as a function of temperature is likely due to thermal expansion. Some of the more rounded features are possibly carbon contamination. In oxygen those features are not observed at the highest temperature where carbon is expected to oxidize.

A morphology variation is observed for the sample in oxygen. At $400{ }^{\circ} \mathrm{C}$ the LSC crystal structure appears to break up into a fine nanograin structure, which becomes more visible at $600{ }^{\circ} \mathrm{C}$ (indicated by white arrows). A similar nanograin structure is barely visible in vacuum even at $600{ }^{\circ} \mathrm{C}$. These observations are consistent with our previous observations [18] and indicate that the LSC stability decreases with increasing oxygen pressure at elevated temperatures.

According to Fig. 2, only minor morphological changes are induced by heating, both vacuum and oxygen. We will now focus on changes in chemistry as a response to the change in temperature.

Fig. 3 presents two different ways to analyze Co oxidation states. The Co white line intensity ratio can be used as an indicator of the Co oxidation state [23]. Fig. 3a presents white line ratios at different temperatures for LSC1 and LSC2 in the symmetric model cells exposed to oxygen or vacuum. Known intensity ratios obtained from standards with specific Co oxidation states are indicated by dark grey areas [23,24]. According to Fig. 3a, the oxidation state for Co in LSC increases for increasing temperature above $400-500{ }^{\circ} \mathrm{C}$.

Fig. $3 \mathrm{~b}$ shows the energy difference between the Co- $\mathrm{L}_{3}$ and $\mathrm{Co}-\mathrm{L}_{2}$ white lines for LSC in oxygen and in vacuum as a function of temperature. Measurement errors for edge energy differences are based on the estimated accuracy of the fitting procedure. There is some scattering of the data points, but the overall trend in both gas environments is a decreasing energy difference for increasing temperature above ca. $400-500{ }^{\circ} \mathrm{C}$ from ca. $15.3 \mathrm{eV}$ to $14.8 \mathrm{~V}$. A previous study correlated a decreasing energy difference from $15.4 \mathrm{eV}$ to $14.5 \mathrm{eV}$ to an increase in the Co oxidation state from $\mathrm{Co}^{2+}$ to $\mathrm{Co}^{3+}[17]$.

Both the analysis by white line intensity ratio (Fig. 3a) and the analysis by relative Co peak energy differences (Fig. 3b) leads to the same conclusion of increasing Co oxidation state as a function of temperature above $400-500{ }^{\circ} \mathrm{C}$. This means that $\mathrm{Co}$ is oxidized at temperatures above $400-500{ }^{\circ} \mathrm{C}$, not only in an oxygen atmosphere, but also in high vacuum.

Fig. 4 shows $\mathrm{Co}_{-} \mathrm{L}_{2,3}$ and $\mathrm{O}-\mathrm{K}$ edges at various temperatures for LSC in vacuum and oxygen. The spectra are generally similar to previously reported EELS measurements [25-27].

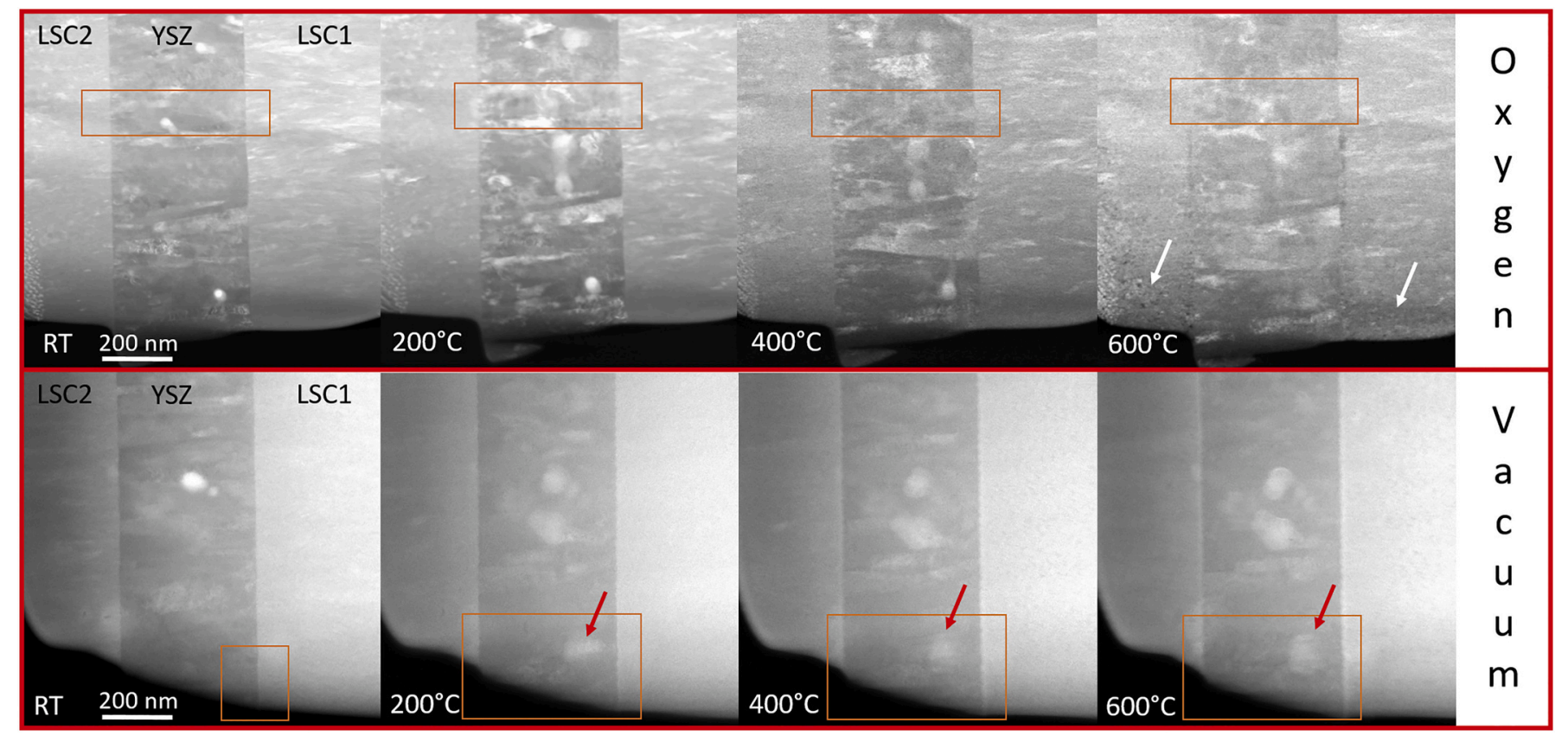

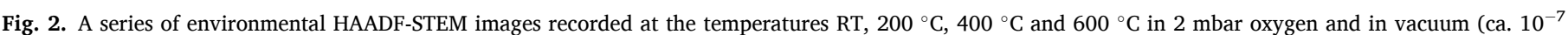

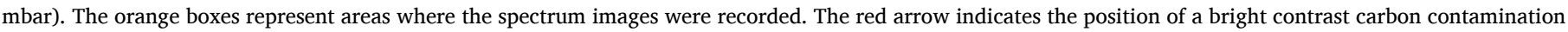

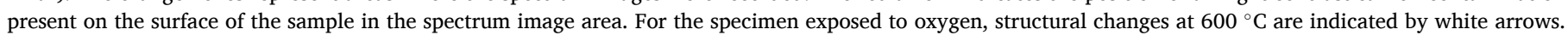



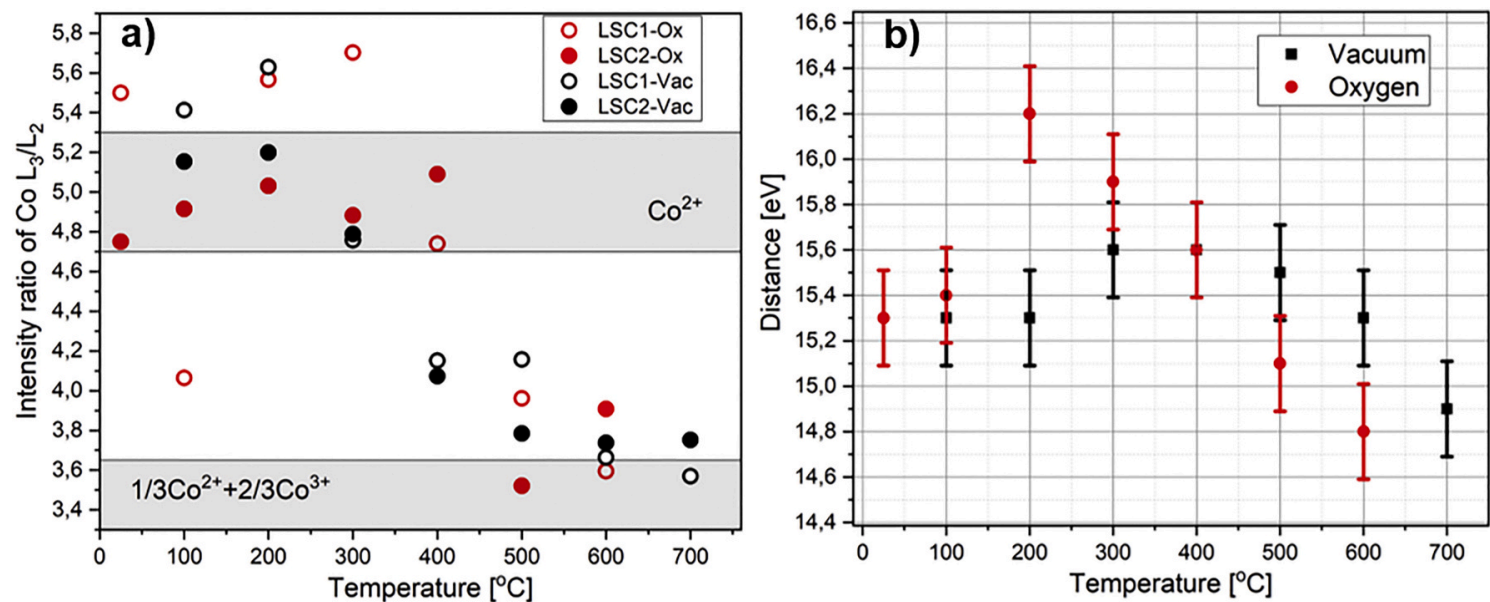

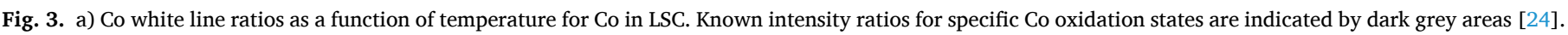

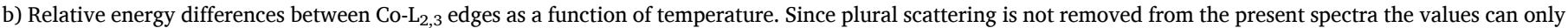
be approximately compared to those in the grey areas and it is more the trend in data that is important than the absolute values.
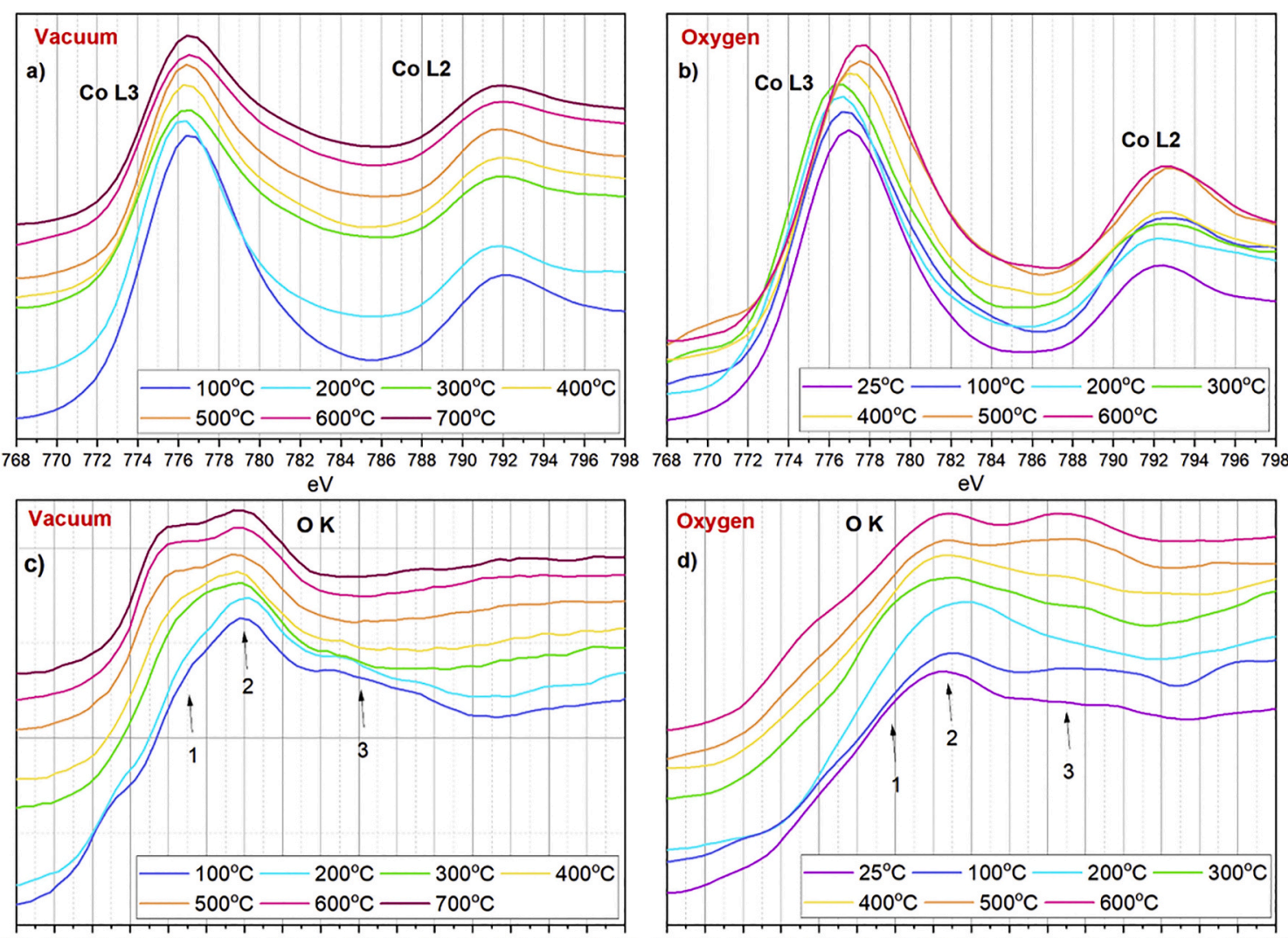

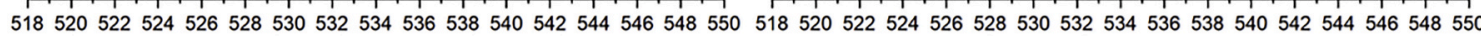
$\mathrm{eV}$

$\mathrm{eV}$

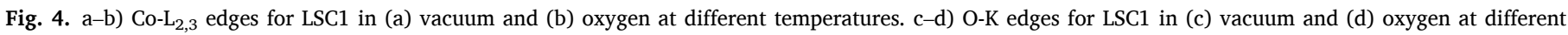
temperatures. (a) and (b) present data used for the analysis presented in Fig. 3.

For the Co- $\mathrm{L}_{3,2}$ edges, increasing the temperature both in vacuum and oxygen leads to broadening (fig. $4 a-b$ ) which can indicate an increase in oxidation state for transmission metals [15,23]. In perovskite compounds, the O-K edge fine structure generally form three main peaks $[15,28,29]$ shown by numbers 1,2 and 3 in Fig. $4 c-d$. Peak 1 identifies excitation from $\mathrm{O} 1 \mathrm{~s}$ electrons to $2 \mathrm{p}$ unoccupied states hybridized with Co 3 states, while peak 2 identifies hybridization with $\mathrm{Sr} 4 \mathrm{~d}$ and La 5d states and peak 3 with Co $4 \mathrm{~s}$ and 4p states, respectively [29-31].

Fig. 4 shows that in vacuum, peak 1 increases with temperature, while the others remain constant. An increase in the first peak indicate oxidation [15]. In oxygen, on the other hand, only peak 3 increases with temperature, which could indicate oxidation of other transition metals than Co [29]. It can therefore be speculated that LSC near YSZ in oxygen partly transforms into other compounds involving La and Sr.

It should be noticed that both in oxygen and high vacuum and for all temperatures, analysis across the specimen of the backgroundsubtracted edge intensities did not indicate segregation of La, Sr, Co, Y or $\mathrm{Zr}$ (not presented). 


\section{Discussion}

When $\mathrm{Sr}^{2+}$ is introduced to substitute $\mathrm{La}^{3+}$ cations in LSC the excess of negative charge due to its lower valence is compensated by oxidation of $\mathrm{Co}^{3+}$ to $\mathrm{Co}^{4+}$ and creation of oxygen vacancies as proposed firstly by Jonker and van Santen [32].

According to Fig. 3, at room temperature Co has a lower oxidation state than expected for the bulk LSC, apparently close to $\mathrm{Co}^{2+}$. This is the case both in high vacuum and in 2 mbar oxygen. However, since plural scattering is not removed from the present spectra the values can only be approximately compared to reference values and the error on the oxidation state is difficult to estimate. The observation of such a low oxidations state is not unique. For other perovskites, low cation oxidation states have been reported at grain boundaries compared to bulk $[14,15] . \mathrm{Co}^{2+}$ has previously been reported for LSC with La-to-Sr ratio = 1. When elastic stress is applied high concentrations of oxygen vacancies in LSC have been reported [23,33-36]. Wang et al. found a Co $\mathrm{L}_{3}-\mathrm{to}_{-} \mathrm{L}_{2}$ intensity ratio of ca. 5 [23], similar to that observed for Co in our model cell (Fig. 3a).

A possible explanation for a low Co oxidation state in the present model cell could be related to strain. The strain in thin films has been associated this high oxygen deficiency $[34,37]$. Similarly, mismatch between the present LSC and YSZ could lead to stress or strain leading to an increased concentration of oxygen vacancies and therefore to the observation of Co oxidation states below expected values for bulk LSC at room temperature (Fig. 3a).

When increasing the temperature above $400-500{ }^{\circ} \mathrm{C}$, the oxidation state of Co in LSC increases (Fig. 3). There are different possible explanations for this.

Firstly, if this was only observed in oxygen atmosphere, this could suggest that LSC is oxidized by the gas phase oxygen. However, since the same trend is observed in vacuum, the results more likely suggest that oxygen is transported from within the materials when the temperature allow for sufficient oxygen ion conductivity.

Secondly, once the structure reaches a temperature close to the one used during the deposition of the thin film by PLD $\left(450^{\circ} \mathrm{C}\right)$, the strain can be relaxed, and re-oxidation of $\mathrm{Co}^{2+}$ to $\mathrm{Co}^{3+}$ occurs in the strained areas.

A third possible explanation is that the observed oxidation is a result of LSC decomposition. Instability of LSC in reducing environments or a low oxygen partial pressure at high temperature is known, and when the temperature becomes high enough, decomposition effects start to play a role [18]. This explanation is consistent with the discussion of the changes in the O-K edge fine structure and with the morphology changes at the higher temperatures commented on in relation to Fig. 2. The later applies best to the case in oxygen, and less to the case in vacuum where the morphological changes were less pronounced.

The present results relate to the concept of "nanoionics", a term that was coined to identify ionic conductors with nanometric features different from that of bulk materials [38-40]. The vicinity of heterojunctions can have a space charge layer (SPL) or lattice strain effects, leading to increase in the density of ionized species such as oxygen vacancies [41] and to superionic conductivity [42].

For the present system, the Co oxidation state approach bulk-like characteristics for temperatures where SOFCs/SOECs are typically operated (Fig. 3a). This means that the possible advantage of a nanoionic high conductivity effect across the electrode-electrolyte interface minimizes or even disappears at these high temperatures.

The present study also highlights the importance of studying possible nanoionics effects in situ since the chemistry (Figs. 3-4) is shown to be sensitive to temperature variations and the gas environment influences morphology at the nanoscale (Fig. 2).

\section{Conclusion}

LSC-YSZ interfaces in a symmetric model SOEC/SOFC was analysed by environmental STEM-EELS in high vacuum and in oxygen at elevated temperatures. The crystalline specimen was grown as by PLD, but at temperatures above $400^{\circ} \mathrm{C}$, a fine grain morphology was observed at the LSC while the YSZ was apparently unaffected. EELS analysis indicates that the oxidation state of Co in LSC near an LSC-YSZ interface is lower than $\mathrm{Co}^{4+}$ expected for bulk LSC. However, the oxidation state for Co increases as the temperature was raised above $400-500{ }^{\circ} \mathrm{C}$, both in oxygen and vacuum, and the oxidation state of Co approached the bulk value when the temperature approach typical operation temperatures for solid oxide electrolysis and fuel cells. This offers a possible explanation for reasonable good predictions of experimental results based on bulk values, despite observations of nanoionics effects at room temperature.

\section{Declaration of Competing Interest}

The authors declare that they have no known competing financial interests or personal relationships that could have appeared to influence the work reported in this paper.

\section{Acknowledgement}

We gratefully acknowledge the financial support of this research from In Situ Transmission Electron Microscopy on Operating Electrochemical Cells (TEMOC) project, supported by the Danish Council for Independent Research grant no. DFF - 4005-00247. The A P Møller and Christine Mc-Kinney Møller Foundation are gratefully acknowledged for their contribution towards the establishment of the Center for Electron Nanoscopy in the Technical University of Denmark. Part of this work was supported by Nagoya University microstructural characterization platform as a program of "Nanotechnology Platform" of the Ministry of Education, Culture, Sports, Science and Technology (MEXT), Japan.

\section{References}

[1] V. Dusastre, J.A. Kilner, Optimisation of composite cathodes for intermediate temperature SOFC applications, Solid State Ionics 126 (1999) 163-174, https:// doi.org/10.1016/S0167-2738(99)00108-3.

[2] P. Möller, R. Kanarbik, I. Kivi, G. Nurk, E. Lust, Influence of microstructure on the electrochemical behavior of LSC cathodes for intermediate temperature SOFC, J. Electrochem. Soc. 160 (2013) F1245-F1253, https://doi.org/10.1149/ 2.037311jes.

[3] K. Develos-Bagarinao, J. De Vero, H. Kishimoto, T. Ishiyama, K. Yamaji, T. Horita, H. Yokokawa, Multilayered LSC and GDC: an approach for designing cathode materials with superior oxygen exchange properties for solid oxide fuel cells, Nano Energy 52 (2018) 369-380, https://doi.org/10.1016/j.nanoen.2018.08.014.

[4] I. Garbayo, V. Esposito, S. Sanna, A. Morata, D. Pla, L. Fonseca, N. Sabaté, A. Tarancón, Porous $\mathrm{La}_{0.6} \mathrm{Sr}_{0.4} \mathrm{CoO}_{3-\delta}$ thin film cathodes for large area micro solid oxide fuel cell power generators, J. Power Sources 248 (2014) 1042-1049, https:// doi.org/10.1016/j.jpowsour.2013.10.038.

[5] J. Januschewsky, M. Ahrens, A. Opitz, F. Kubel, J. Fleig, Optimized $\mathrm{La}_{0.6} \mathrm{Sr}_{0.4} \mathrm{CoO}_{3-\delta}$ thin-film electrodes with extremely fast oxygen-reduction kinetics, Adv. Funct. Mater. 19 (2009) 3151-3156, https://doi.org/10.1002/ adfm.200900362.

[6] M. Backhaus-Ricoult, SOFC - a playground for solid state chemistry, Solid State Sci. 10 (2008) 670-688, https://doi.org/10.1016/j.solidstatesciences.2007.11.021.

[7] A.N. Petrov, O.F. Kononchuk, A.V. Andreev, V.A. Cherepanov, P. Kofstad, Crystal structure, electrical and magnetic properties of $\mathrm{La}_{1-\mathrm{x}} \mathrm{Sr}_{\mathrm{x}} \mathrm{CoO}_{3-\mathrm{y}}$, Solid State Ionics 80 (1995) 189-199, https://doi.org/10.1016/0167-2738(95)00114-L.

[8] Yu Chen, S.B. Adler, Thermal and chemical expansion of Sr-doped lanthanum cobalt oxide $\left(\mathrm{La}_{1}\right.$ - $\mathrm{Sr}_{\mathrm{x}} \mathrm{CoO}_{3}$ - $\delta$ ), Chem. Mater. 17 (2005) 4537-4546, https://doi. org/10.1021/cm050905h.

[9] A.N. Petrov, V.A. Cherepanov, A.Y. Zuev, Thermodynamics, defect structure, and charge transfer in doped lanthanum cobaltites: an overview, J. Solid State Electrochem. 10 (2006) 517-537, https://doi.org/10.1007/s10008-006-0124-0.

[10] J. Ovenstone, J.S. White, S.T. Misture, Phase transitions and phase decomposition of $\mathrm{La}_{1-\mathrm{x}} \mathrm{Sr}_{\mathrm{x}} \mathrm{CoO}_{3-\delta}$ in low oxygen partial pressures, J. Power Sources 181 (2008), https://doi.org/10.1016/j.jpowsour.2008.03.033.

[11] J. Mizusaki, Y. Mima, S. Yamauchi, K. Fueki, Nonstoichiometry of the perovskitetype oxides $\mathrm{La}_{1-\mathrm{x}} \mathrm{Sr}_{\mathrm{x}} \mathrm{CoO}_{3-\delta}$, J. Solid State Chem. 80 (1989) 102-111.

[12] J. Mizusaki, Y. Mima, S. Yamauchi, K. Fueki, Nonstochiometry of the perovskite-

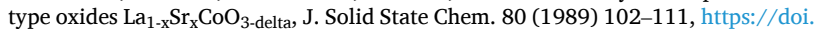
org/10.1002/j.1538-7305.1924.tb01347.x.

[13] T. Kawada, J. Suzuki, M. Sase, A. Kaimai, K. Yashiro, Y. Nigara, J. Mizusaki, K. Kawamura, H. Yugami, Determination of oxygen vacancy concentration in a thin 
film of $\mathrm{La}_{0.6} \mathrm{Sr}_{0.4} \mathrm{CoO}_{3-\delta}$ by an electrochemical method, J. Electrochem. Soc. 149 (2002), E252, https://doi.org/10.1149/1.1479728.

[14] N.D. Browning, J.P. Buban, H.O. Moltaji, S.J. Pennycook, G. Duscher, K. D. Johnson, R.P. Rodrigues, V.P. Dravid, The influence of atomic structure on the formation of electrical barriers at grain boundaries in $\mathrm{SrTiO}_{3}$, Appl. Phys. Lett. 74 (1999) 2638-2640, https://doi.org/10.1063/1.123922.

[15] R.F. Klie, Y. Ito, S. Stemmer, N.D. Browning, Observation of oxygen vacancy ordering and segregation in Perovskite oxides, Ultramicroscopy. 86 (2001) 289-302, https://doi.org/10.1016/S0304-3991(00)00120-0.

[16] R.F. Egerton, Electron energy-loss spectroscopy in the electron microscope, Springer US, 2011, https://doi.org/10.1007/978-1-4419-9583-4.

[17] P. Müller, M. Meffert, H. Störmer, D. Gerthsen, Fast mapping of the cobalt-valence state energy loss spectroscopy, Microsc. Microanal. 19 (2013) 1595-1605.

[18] F. Gaulandris, S.B. Simonsen, J.B. Wagner, S. Sanna, S. Muto, L.T. Kuhn, In situ TEM analysis of a symmetric solid oxide cell in oxygen and vacuum - cation diffusion observations, ECS Trans. 75 (2017) 123-133, https://doi.org/10.1149/ 07542.0123ecst.

[19] T. Hansen, J. Wagner, J. Jinschek, R. Dunin-Borkowski, The titan environmental transmission electron microscope: specifications, considerations and first results, Microsc. Microanal. 15 (2009) 714-715, https://doi.org/10.1017/ S1431927609097396.

[20] D.H. Pearson, C.C. Ahn, B. Fultz, White lines and d-electron occupancies for the 3d and 4d transition metals, Phys. Rev. B 47 (1993) 8471-8478, https://doi.org/ 10.1103/PhysRevB.47.8471.

[21] V.V. Sychugov, P.N. Degtyarenko, A.V. Ovcharov, S.V. Shavkin, V.S. Kruglov, A. L. Vasiliev, P.V. Volkov, Y.M. Chesnokov, Variation of critical current and n-value of $2 \mathrm{G}$ HTS tapes in external magnetic fields of different orientation, J. Phys. Conf. Ser. 747 (2016), https://doi.org/10.1088/1742-6596/747/1/012048.

[22] M. Lao, R. Willa, A. Meledin, H. Rijckaert, V. Chepikov, S. Lee, V. Petrykin, I. Van Driessche, A. Molodyk, B. Holzapfel, J. Hänisch, In-field performance and flux pinning mechanism of pulsed laser deposition grown $\mathrm{BaSnO}_{3} / \mathrm{GdBa}_{2} \mathrm{Cu}_{3} \mathrm{O}_{7-\delta}$ nanocomposite coated conductors by SuperOx, Supercond. Sci. Technol. 32 (2019), 094003, https://doi.org/10.1088/1361-6668/ab2a95.

[23] Z.L. Wang, J.S. Yin, Cobalt valence and crystal structure of $\mathrm{La}_{0.5} \mathrm{Sr}_{0.5} \mathrm{CoO}_{2.25}$, Philos. Mag. B 77 (1998) 49-65, https://doi.org/10.1080/014186398259842.

[24] Z.L. Wang, J.S. Yin, Y.D. Jiang, EELS analysis of cation valence states and oxygen vacancies in magnetic oxides, Micron. 31 (2000) 571-580, https://doi.org/ 10.1016/S0968-4328(99)00139-0.

[25] W.G. Waddington, P. Rez, I.P. Grant, C.J. Humphreys, White lines in the L2,3 electron-energy-loss and x-ray absorption spectra of 3d transition metals, Phys. Rev. B 34 (1986) 1467-1473, https://doi.org/10.1103/PhysRevB.34.1467.

[26] P.E. Vullum, A.T.J. Van Helvoort, R. Holmestad, J. Mastin, Ø.E. Andersen, M. A. Einarsrud, T. Grande, Grain boundary analysis and secondary phases in LaCoO3based perovskites, J. Mater. Sci. 42 (2007) 6267-6273, https://doi.org/10.1007/ s10853-006-1252-8.

[27] H.-I. Ji, J. Hwang, K.J. Yoon, J.-W. Son, B.-K. Kim, H.-W. Lee, J.-H. Lee, Enhanced oxygen diffusion in epitaxial lanthanum-strontium-cobaltite thin film cathodes for micro solid oxide fuel cells, Energy Environ. Sci. 6 (2013) 116, https://doi.org/ $10.1039 / \mathrm{c} 2 \mathrm{ee} 21647 \mathrm{~g}$

[28] Y. Ito, R.F. Klie, N.D. Browning, T.J. Mazanec, Atomic resolution analysis of the defect chemistry and microdomain structure of brownmillerite-type strontium cobaltite, J. Am. Ceram. Soc. 85 (2002) 969-976, https://doi.org/10.1111/j.11512916.2002.tb00201.x

[29] M. Abbate, F.M.F. De Groot, J.C. Fuggle, A. Fujimori, O. Strebel, M.F. Lopez, M. Domke, G. Kaindl, G.A. Sawatzky, M. Takano, Y. Takeda, H. Eisaki, S. Uchida, Controlled-valence properties of $\mathrm{La}_{1-\mathrm{x}} \mathrm{Sr}_{\mathrm{x}} \mathrm{FeO}_{3}$ and $\mathrm{La}_{1-\mathrm{x}} \mathrm{Sr}_{\mathrm{x}} \mathrm{MnO}_{3}$ studied by soft-xray absorption spectroscopy, Phys. Rev. B 46 (1992) 4511-4519, https://doi.org/ 10.1103/PhysRevB.46.4511.

[30] J. Verbeeck, O.I. Lebedev, G. Van Tendeloo, J. Silcox, B. Mercey, M. Hervieu, A. M. Haghiri-Gosnet, Electron energy-loss spectroscopy study of a $\left(\mathrm{LaMnO}_{3}\right)_{8}\left(\mathrm{SrMnO}_{3}\right)_{4}$ heterostructure, Appl. Phys. Lett. 79 (2001) 2037-2039, https://doi.org/10.1063/1.1403316.

[31] B. Han, K.A. Stoerzinger, V. Tileli, A.D. Gamalski, E.A. Stach, Y. Shao-Horn, Nanoscale structural oscillations in perovskite oxides induced by oxygen evolution, Nat. Mater. 1 (2016), https://doi.org/10.1038/nmat4764.

[32] G.H. Jonker, J.H. Van Santen, Magnetic compounds wtth perovskite structure III. ferromagnetic compounds of cobalt, Physica 19 (1953) 120-130, https://doi.org/ 10.1016/S0031-8914(53)80011-X.

[33] M. Varela, J. Gazquez, S.J. Pennycook, STEM-EELS imaging of complex oxides and interfaces, MRS Bull. 37 (2012) 29-35, https://doi.org/10.1557/mrs.2011.330.

[34] J. Gazquez, W. Luo, M.P. Oxley, M. Prange, M.A. Torija, M. Sharma, C. Leighton, S. T. Pantelides, S.J. Pennycook, M. Varela, Atomic-resolution imaging of spin-state superlattices in nanopockets within cobaltite thin films, Nano Lett. 11 (2011) 973-976, https://doi.org/10.1021/nl1034896.

[35] J. Gázquez, G. Sánchez-Santolino, N. Biškup, M.A. Roldán, M. Cabero, S. J. Pennycook, M. Varela, Applications of STEM-EELS to complex oxides, Mater. Sci. Semicond. Process. 65 (2017) 49-63, https://doi.org/10.1016/j. mssp.2016.06.005.

[36] S. Stemmer, A.J. Jacobson, X. Chen, A. Ignatiev, Oxygen vacancy ordering in epitaxial $\mathrm{La}_{0.5} \mathrm{Sr}_{0.5} \mathrm{CoO}_{3-\delta}$ thin films on (001) LaAlO3, J. Appl. Phys. 90 (2001) 3319-3324, https://doi.org/10.1063/1.1401793.

[37] T. Nakamura, K. Yashiro, T. Kawada, J. Mizusaki, Evaluation of high-temperature electronic and electrochemical properties of the strained $\mathrm{La}_{1-\mathrm{x}} \mathrm{Sr}_{\mathrm{x}} \mathrm{CoO}_{3-\delta}$ films prepared by a pulsed laser deposition technique, Electrochemistry. 82 (2014) 884-890, https://doi.org/10.5796/electrochemistry.82.884.

[38] J. Schoonman, Nanoionics, Solid State Ionics 157 (157) (2003) 319-326.

[39] J. Maier, Ionic transport in nano-sized systems, Solid State Ionics 175 (2004) 7-12, https://doi.org/10.1016/j.ssi.2004.09.051.

[40] J. Maier, Pushing nanoionics to the limits: charge carrier chemistry in extremely small systems, Chem. Mater. 26 (2014) 348-360, https://doi.org/10.1021/ cm4021657.

[41] J. Maier, Defect chemistry and ionic conductivity in thin films, Solid State Ionics 23 (1987) 59-67, https://doi.org/10.1016/0167-2738(87)90082-8.

[42] J. Maier, Nanoionics: ion transport and electrochemical storage in confined systems, Nat. Mater. 4 (2005) 805-815. 